\title{
Carnets
}

Revue électronique d'études françaises de l'APEF

Deuxième série - 13 | 2018

Corps, rythmes et voix : en/jeux littéraires et artistiques

\section{Faire craquer l'épidermico-sémantique}

Voix, performance et travail du négatif chez Christian Prigent

\section{Guilherme Massara Rocha}

\section{CpenEdition}

\section{Journals}

Édition électronique

URL : http://journals.openedition.org/carnets/2639

DOI : $10.4000 /$ carnets.2639

ISSN : 1646-7698

Éditeur

APEF

Référence électronique

Guilherme Massara Rocha, «Faire craquer l'épidermico-sémantique », Carnets [En ligne], Deuxième série - 13 | 2018, mis en ligne le 31 mai 2018, consulté le 22 avril 2019. URL : http:// journals.openedition.org/carnets/2639; DOI : 10.4000/carnets.2639

Ce document a été généré automatiquement le 22 avril 2019

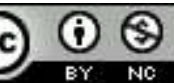

Carnets est mis à disposition selon les termes de la licence Creative Commons - Atribution - Pas d'utilisation commerciale 4.0 International. 


\title{
Faire craquer l'épidermico- sémantique
}

\author{
Voix, performance et travail du négatif chez Christian Prigent
}

Guilherme Massara Rocha

1 La poésie de Christian Prigent semble atteindre sa plus grande expressivité esthétique dans une activité de lecture que l'artiste appelle «performance sonore» (Prigent, 2004 : 35) ou "performance orale». En lisant des extraits de ses écrits, Prigent conduit l'auditeur à une expérience de contraposition artistique entre la flamme intérieure et la sobriété extérieure de sa verve narrative. La posture corporelle statique n'est interrompue que par un mouvement particulier de sa main droite, qui semble suivre le rythme d'une certaine cadence des syllabes, et qui sert au mouvement du phrasé. La cadence non linéaire de ses exercices de lecture est accompagnée de suaves mouvements de pied, qui font penser aux exercices musicaux de temporalisation des échelles ou des harmonies. Des pieds qui marquent le rythme, ou les ordres de développement temporel de cette inquiétante orchestralité prosodique caractéristique des performances orales de Prigent.

2 Cependant, la notion de performance orale, terme de prédilection de l'auteur pour désigner de tels exercices de lecture, demande un effort d'élucidation. Le concept esthétique de performance, dès ses premiers usages dans les dernières années de 1950, rassemblait de nombreuses actions individuelles ou collectives dont le but devait déplacer le geste esthétique de ses dispositifs et supports habituels: de la performance résulte ainsi une certaine dématérialisation de l'objet esthétique, qui serait d'ores et déjà conçu comme évanescent, ponctuel et transitoire. Joseph Beuys, Vito Acconti, Bruce Nauman, Marina Abramovic ou Flávio de Carvalho, tous ces artistes sont les protagonistes des performances devenues paradigmatiques et fondées sur des actions corporelles imprégnées de symbolisme, dont l'enjeu consiste souvent à interroger les sens socialement partagés d'une expérience. «Dans son essence la performance prétend ne pas avoir un medium, tout en diffusant des informations en direct et sans aucune entremise » (Ruhrberg et al., 2005 : 601) Beuys, pendant trois semaines, cohabite dans une cage avec un coyote sauvage. Il y gesticule et manipule des tissus, dans I like America and 
America likes me, et il se sert d'une ambulance pour rentrer et pour sortir du pays. Fréquemment nus, Abramovic et Ulay performent des mouvements, actions et interactions répétitives, jusqu'aux limites de l'épuisement physique. Flávio de Carvalho, à la fin des années 1940, marche en direction contraire d'une procession Corpus Christi à São Paulo, et sans enlever son chapeau. Après avoir échappé à un lynchage grâce aux gendarmes, il écrit un livre Experiência $n .2$ où il interprète, d'après des concepts esthétiques et psychanalytiques, les réactions haineuses des fidèles.

Dans ces types de performance on retrouve un appel politique qui est mis en place tantôt par des ironies subtiles, tantôt par des protocoles formels qui mettent en scène plus directement le corps, (la nudité, la violence, le comportement profane). On remarque la façon dont la performance est un dispositif esthétique fréquemment cernée par la tension entre le discours et l'acte. Ayant recours à la performativité narrative ou expressive de l'acte, la performance se produit souvent sans avoir recours au signifiant, et se sert justement des abstractions de l'expression verbale pour toucher le sens ou les sens de l'expérience. Sans l'obligation de véhiculer des significations, l'acte performatif voltige en rasant le sol et les ruissellements du sensible, excitant le réel et convoquant ses effets. Dans ce sens, un des aspects des plus énigmatiques des performances de Prigent est justement une suppression de l'antithèse dialectique entre l'acte et le discours. Ce dispositif est justement le "phrasé ». Il dit :

(...) c'est la forme du texte qui fait sens. Ou est dans un certain effet de phrasé, dans le rythme, la vitesse et la condensation de ce phrasé que résident la portée et le sens d'un geste d'écriture. Parce que le sens de ce geste (ce qui l'impulse, ce qui le modèle, ce qu'il cherche à produire comme effet) s'incarne dans une puissance rythmique d'arrachement au lieu commun et dans la proposition d'une vision inouïe, adéquate à la singularité de l'expérience (Prigent, 2009 : 42)

4 Le privilège donné ici à la forme du texte semble ne pas se confondre avec un formalisme calculé, à la façon des exercices de la poésie concrète, où il y a une visée d'expansion du circuit de l'appréhension des significations malgré la syntaxe abrégée ou minimaliste. Rythme, vitesse et condensation sont les protocoles formels de Prigent. La condensation la tension interne de l'écriture entre le non-sens et les couches superposées de signification - est le résultat, dans sa performance orale, de l'acte phonologique fondé sur le rythme et la vitesse. Mais elle est également fondée sur les inflexions vocales qui n'ont guère la fonction d'ajouter au texte des couches supplémentaires de sens (ou d'affection), comme dans les cas où un texte écrit est chanté ou soutenu par une mélodie. Prigent travaille notamment du côté de l'extraction, il procède en exposant les ruissellements de l'écrit, sa matérialité paradoxale ou son substrat sonore. Ce sont les voix qui se font présentes au cours de ses performances orales, «la voix poétique comme espacement scandé et évidemment soufflé de l'enchaînement : comme fendu déchaîné dans le fondu enchaîné des traits qui articulent le sens » (Prigent, 2004 : 35).

Arthur Danto argumente que la "partie arbitraire de l'objet» (Danto, 2005: 190) sélectionnée par une interprétation esthétique est justement ce qui rend reconnaissable la forme d'une œuvre d'art. Alors, sans une interprétation, soit la forme est à nouveau dans l'objet, soit elle disparait. L'effet de l'interprétation, rendu indissociable de la réalisation expressive d'une procédure ou performance artistique se définit, selon Danto, comme un exercice de narration, ou d'exégèse, qui accueille la puissance expressive de l'œuvre d'art dans une nouvelle configuration identitaire. Cependant, chez Prigent, l'arrachement ou la transfiguration du lieu commun d'une écriture artistique suit une interprétation qui se fait présente plutôt comme un acte de violence, et non comme une 
élucidation - d'après Foucault. L'interprétation est à la fois mise en scène et incorporée à l'exercice même de vocalisation du texte, et ainsi, sa tendance non identitaire et sa négativité sont probablement encore renforcées. Celui que témoigne les performances orales de Prigent semble noyé dans un habitat verbal, dans lequel des molécules de sens fusionnent en créant une signification transitoire, qui n'y demeure pas à cause de la vitesse non affectée des syllabes et des syntagmes. Dans ce contexte, le rythme de l'interprétation ne se relève d'aucune adéquation entre le contenu sémantique et les lignes directrices de signification. Quoi qu'il en soit, il n'est pas non plus son opposé, c'est-à-dire une sorte d'artifice illimité de déconstruction ou d'éparpillement de sens. Le noyau de la performance orale est solidaire de ce que Prigent désigne comme la voix du texte, autrement dit «un coefficient de densité opaque et de chaos in-signifiant équivalents de ce que nous propose la vie » (Prigent, 2009 : 250).

Dans la performance orale, le rythme du phrasé produit des scansions dans le texte à partir des effets de désarticulation/ré-articulation, qui sont suscités par ce privilège esthétique de la voix. On pourrait dire, tout en poursuivant une des indications de Mladen Dolar, que dans la performance orale de Prigent «le signifiant s'épuise dans la voix comme son résultat» (Dolar, 2012: 46). Voilà, peut-être, la base positive pour une négativité inhérente à l'écriture qui sous-tend de telles performances : une base positive et fugace, "juste vibrations de l'air qui disparaissent aussitôt qu'elles sont produites " (Dolar, 2012 : 47). Des performances dans lesquelles les frontières de la voix et de ses actes ne sont pas discernables de celles des sens, qui ne se conservent que dans les traits et dans les traces de sa propre disparition.

Écoutons et mortifions : on faute toujours, en vrai ou en songe ou par omission. Et qui sait tout, il sait, ça comme le reste. Et si c'est la manne de rosée qui crache celui qui sait tout, ou son délégué, comme grenadine ou la menthe à l'eau par les meurtrières ou mâchicoulis du donjon des nues, ça va avant tout gadouiller ma raie peignée au milieu et je vois déjà mère qui furibarde. (Prigent, $2007: 22$ )

7 Peut-être peut-on reconnaître dans cette procédure performative entre l'écrit et la voix quelque chose qui s'approche de ce que le philosophe Jacques Rancière appelle la phraseimage. Il s'agit d'un protocole esthétique où « la phrase n'est pas le dicible » et « l'image n'est pas le visible » (Rancière, $2003: 56$ ). La phrase-image représente, donc, l'articulation entre deux fonctions (écriture et image), mais avec la particularité qu'elle « dédouble la force chaotique de la grande parataxe en puissance phrastique de continuité et puissance imageante de rupture ». «Le résultat », dit-il, c'est qu'on « ne voit pas ce que dit ce qu'on voit, ni ce que donne à voir ce qu'on dit » (Rancière, 2003 : 58). Pour préciser le noyau constitutif de la phrase-image, Rancière revendique la notion méta-psychologique et esthétique de montage. Il est bien possible que Prigent, parlant d'une langue que luimême baptise comme le populo-lacanien 11 , fasse naître son activité performative d'une esthétique du montage, qui pourrait être comprise dans le sens lacanien du terme. Dans ses analyses de la théorie pulsionnelle de Freud, Lacan précise la notion de montage pulsionnel comme ce qui introduit dans l'univers des schémas identitaires et autoperceptifs du sujet de l'inconscient un arrangement hétéroclite d'éléments (ou d'objets) d'investissement de la pulsion. Des fragments d'images d'une remarquable densité corporelle, perçus comme des blocs de signifiants (plutôt que des significations), sont disposés dans un arrangement - un montage - d'appel véritablement sensible. Lacan fait remarquer la manière dont ces fragments de représentations sont, à leur tour, irréductibles aux fonctions autoréférentes et synthétiques de l'unité du Moi. Il écrit : 
Je dirai que le montage de la pulsion c'est un montage qui d'abord en apparence se présente pour nous comme n'ayant ni queue ni tête, comme un montage au sens où l'on parle de montage dans un collage surréaliste (...) je crois que l'image qui nous vient c'est je ne sais quoi qui montrerait: 'la marche d'un dynamo qui serait branchée sur la prise du gaz avec quelque part une plume de paon qui en sort et vient chatouiller le ventre d'une jolie femme qui est là à demeure, pour la beauté de la chose' (...) vous y verrez à tout l'instant le saut sans transition des images le plus hétérogènes les unes aux autres. Et tout ceci ne passant que par des références grammaticales dont il vous sera aisé de saisir l'artifice (Lacan, 1964 : 94).

Rappelons que la référence à cette notion de montage pulsionnel renvoie à l'idée de phrase-image de Jacques Rancière. Cependant, Rancière remarque que le montage n'est pas la simple opposition des termes, « où triomphe nécessairement le terme qui donne son ton à l'ensemble» (Rancière, 2003: 59). Ainsi, quand on analyse la négativité en question dans l'esthétique performative de Prigent, il faudrait remarquer que son processus de montage retient vraiment quelque chose de la conceptualisation lacanienne. Elle met en œuvre, plus précisément, une sorte de satisfaction - de jouissance non épuisable pour l'ensemble des représentations - et qui se juxtapose à la dimension réelle des objets dans l'expérience du phrasé. Cette satisfaction est pourtant modalisée d'après les protocoles formels et d'expression que l'écrivain travaille à partir d'un exercice de vocalisation de la matérialité de ces objets. En d'autres termes, le caractère contingent de l'arrangement entre le contenu signifiant et les processus de vocalisation ne doit pas être compris comme un jeu de l'imagination sans contraintes et subordonné à la dispersion pure du hasard. Dans un texte critique intitulé Une erreur de la nature, Prigent semble vouloir faire en sorte que la littérature arrive à la condition d' " ectoplasme verbal qui appâte la lecture et la renvoie, dépitée, à la carence de ses cadences, au précipité aqueux de sa propre précipitation » (Prigent, $1996: 41$ ).

Mais pire c'est pas ça. Pire c'est fauve mauve dans les violacés qui violent ton pensé. Pire s'hérisse en poils de balai farouche pas doux pour le derme. Pire émane merde (...) Pire ricane hi-hi ou hulule funèbre en souille insalubre. Pire a le rut bref sans préliminaires et brut de foutrage. Pire a pas de lit (Prigent, $2003: 64$ )

La voix du texte, noyau expressif de la performance orale, exige de l'interprète la discipline d'une cadence qui « dans le texte, relève des traces de l'oralité implicite qui est l'une des forces qui en ont commandé l'écriture » (Prigent, 2009 : 242). Il y a fréquemment dans ses performances orales quelque chose de drôle ou maladroit, ou même l'occurrence d'un lyrisme fugace, timide, ou mélancolique. Ces évènements, dans les écrits de Prigent aussi, constituent habituellement la face d'une surface rugueuse et polyphonique, où la majorité du motif contre-identitaire du phrasé se retrouve. «Prédilection pour une sorte de recto tono obtus, austère et brutal » (Prigent, 2009: 243). Expressément privé de toute affection psychologique déterminé, le recto tono de Prigent ne peut pas être dit complètement indifférent ou indifférencié.

Père, fais attention, sinon dans bientôt tu vas être mort (...) Il souffle ceci, à peine si j'entends : 'Hier j'étais né, demain je meurs'. Puis regarde ses ongles, referme ses paupières : adieu. Rien d'autre qu'écho, mais écho c'est moi, répète le mot. Il dort. Tu sors en glissé sur la pointe des pieds. Fin de la visite, bientôt fin de tout. Après, tourne talon, air, odeurs du faubourg, le dehors banal. Renfourche le biclou, avale le bitoume, pédale machinal (...) Puis la suite sans pause dans la nuit dehors qui salope tout et oint ta casquette de sa suie de deuil car soir est tombé sur tout le parage et le rideau pareil sur le dernier acte ou l'avant-dernier. Tu pédales à fond dans les encablures, tu re-remoulines plein pot le cerveau ce qu'il t'a glissé comme dernier 
mot dans le tuyau et tu te répètes: viens jamais, demain, viens jamais (Prigent, 2007 : 364-365). une réfraction des protocoles de désaffection et d'impersonnalisation. Il reflète également un moment de l'écrit du sens et des sens qui empêche toute " formalisation apathique ». Voilà un moment où la phrase s'impose sur le phrasé («rythme, écholalie, respiration»), bloquant encore la performance comprise telle une "mise en voix, sensoriellement et formellement possible» (Prigent : 2009 : 245). Dans une formulation lumineuse, Prigent affirme que la fonction de la phrase dans l'écrit est d'être comme "partenaire, comme sparring-partner ", elle éclaire ainsi l'endroit du "seul personnage mis en scène » de ses performances : «le couple bagarreur phrase/phrasé » (Prigent, 2009 : 245). En Météo des Plages, Prigent écrit :

MOI : ce poème est un sublimateur d'éclat (une

Pure brilliance hyperhydratante pour les

Pauvres sites) ; il tend et raffermit chacune

Des leurs surfaces, il rend à la fois l'objet

Net et souple, soyeux étincelant vivement

Coloré - il a enfin bonne mine, on comprend

Où il s'arrête, commence, comment son

Sens s'active à ces energizing nutritions, non ?

En se servant du jargon populo-lacanien, on ajoute que la performance orale de Prigent extrait de la surface des juxtapositions signifiantes la primauté de la lettre, de même qu'elle retire des écarts et de la richesse des images la fonction pulsionnelle du regard. La lettre et le regard semblent y remplir la fonction paradoxale qui est de rendre plus tendues les extrémités du discours, en bouleversant - pour la beauté de la chose - « des modes de la parole, des (in)formes de visibilité et des protocoles d'intelligibilité " (Rancière, 2003 : 85). Dans un autre essai, Rancière affirme que l'expérience narrative contemporaine est marquée par la présence de certains détails insensés, inconditionnés et inutiles et sa fonction serait tout simplement de dire « je suis le réel », le réel qui «n'y a pas besoin pour être là d'avoir une raison d'y être ». Voilà ce que le philosophe appelle «l'évidence sans phrase du réel» (Rancière, 2014: 19). Alors, cette illisibilité presque imminente qu'on atteste dans l'expérience d'écriture et d'oralité chez Prigent est aussi la condition même de son existence. Elle détermine une place paradoxale où les affections réciproques entre le corps et la parole peuvent être vécues.

Bénédicte Gorrillot, qui travaille et qui fait travailler la littérature de Christian Prigent (à partir de ses études, mais surtout à partir des questions et des provocations posées à l'auteur dans de longs entretiens et débats), affirme que «l'(il)lisibilité renverrait à un seuil. Certes le seuil est lieu, un lieu différentiel que marque un saut et qui fait limite, mais c'est un lieu infime, impossible à saisir avec certitude - un quasi non-lieu » (Gorrillot \& Lescart, 2014 : 16). Dans le seuil Prigentien, ce qu'il y semble être mis à côté de cette évidence sans phrase du réel c'est un certain réel du phrasé, dont les protocoles incluent le traitement d'un objet fondamental qu'on abordera brièvement pour approcher la conclusion de cet exposé.

[dé/trou/oüir/ire : détrouïre, dit-il]

LUI : coups de dé/bris de vocabulaires, ces

bulles nulles en l'air! MOI : ça vaque au Pré des

Sons ; avec on peint Fresques portatives ou 
Des fanions vifs piqués sur le plein de tout : des trous (Prigent, 2010 :

99).

13 Parmi plusieurs aspects fascinants dans l'œuvre de Christian Prigent, on trouve son impressionnante habileté de penser sa propre écriture. L'ampleur et la précision de ses observations critiques autour de ses écrits sont remarquables. Dans un passage - Je voix double - le poète éclaircit les fondements de sa performance orale.

Une dynamique lancée dans le tas de souvenirs, des fantasmagories, des pensées inquiètes et des ruminations minables pour en jouer l'apparition par flashes et la disparition catastrophiques (...) c'est l'enjeu. La lecture orale à chaque fois la rejoue : tentant de faire ré-apparaître, démonstrativement vivante, cette forme respiratoire (Prigent, $2009: 244)$.

14 Cette forme respiratoire, qui par hasard s'extrait des poussées abstraites du mot, ou des appels psycho-physiques de la sonorité tonale, n'est pas autre chose que ce que Bernard Baas appelle la voix déliée, une voix qui « est donc très exactement ici l'objet qui tombe de l'Autre et qui se manifeste isolément » (Baas, 2010 : 190). Voix qui pousse l'écriture de Prigent en général, et son phrasé en particulier, au-delà des protocoles d'une phraseimage. Mladen Dolar, dans un très beau livre intitulé Une voix et rien d'autre, affirme poétiquement que « la voix est à la fois la forme la plus subtile et la plus perfide de la chair » (Dolar, 2012 : 63). Ainsi, « la voix se présente comme le lien qui relie le signifiant au corps » et elle ajoute au visible et au lisible un miroir sonore au travers duquel on unifie, chez le sujet, ses appels de reconnaissance et d'identité. La voix qui s'impose dans le phrasé de Prigent est, pourtant, au contraire : «la voix [qui] sape toute certitude et tout fondement d'un sens fermé. La voix illimitée, dépourvue de garantie » (Dolar, 2012 : 65). La voix, dans un mouvement alterné d'approximation "et écart de son ancrage textuel, devient insensée et menaçante» (Dolar, 2012: 56), ou vide et frivole, voire séduisante et enivrante.

15 Cependant, cette voix ne pourrait être réduite, comme un certain tournement déconstructiviste pourrait le supposer, à une simple présence en soi. Dans un certain sens, elle semble plus proche de ce que Lacan identifie à l'objet de la pulsion invoquante, une « voix sans corps, le reste, la trace de l'objet» qui «incarne l'impossibilité même d'atteindre l'auto-affection ». Voix qui introduit « une scission, une rupture au milieu de la présence pleine » et qui se pose comme «obstacle intérieur à la présence (à soi) » (Dolar, 2012 : 56). Dans ce sens on est d'accord avec Mladen Dolar quand il affirme que « la voix semble posséder le pouvoir de transformer les mots en actes" et que, dans cette perspective, elle pourrait " représenter la forme originaire de la performativité » (Dolar, 2012 : 71). Voix qui n'est pas du sens ou des sens. Disons-le en populo-lacanien : voix de l'ab-sens ou, comme le remarque Jacques Lacan dans un texte prigentien qui s'appelle l'Étourdit, voix qui fait que ce «qu'on dise reste oublié derrière ce qui se dit dans ce qui s'entend » (Lacan, 2001 : 449). 


\section{BIBLIOGRAPHIE}

BAAS, Bernard (2010). La voix déliée. Paris : Hermann.

DANTO, Arthur (2005). A transfiguração do lugar comum. São Paulo : Cosac Naify.

DOLAR, Mladen (2012). Une voix et rien d'autre. Caen : Nous.

foucault, Michel [1997 (1975)]. Nietzsche, Freud \& Marx. São Paulo : Princípio.

LACAN, Jacques (1964). Le séminaire 11 - les quatres concepts fondementaux de la psychanalyse. [En

ligne] URL : http://staferla.free.fr/S11/S11\%20FONDEMENTS.pdf.

LACAN, Jacques (2001). Autres écrits. Paris : Seuil.

PRIGENT, Christian. (1996). Une erreur de la nature. Paris : P.O.L.

PRIGENT, Christian (2003). Grand-mère Quéquette. Paris : P.O.L.

PRIGENT, Christian (2004). L'Incontenable. Paris : P.O.L.

PRIGENT, Christian (2007). Demain je meurs. Paris : P.O.L.

PRIGENT, Christian (2009). Christian Prigent, quatre temps - rencontre avec Bénédicte Gorrillot. Paris :

Argol.

PRIGENT, Christian (2010). Météo des plages. Paris : P.O.L.

RANCIERE, Jacques (2003). Le destin des images. Paris : La fabrique.

RANCIERE, Jacques (2014). Le fil perdu - essais sur la fiction moderne. Paris : La fabrique.

\section{NOTES}

1. "Mon français 'moderne' est en fait souvent très... ancien. Lexicalement, il s'acommode de n'être que du latin raccourci; syntatiquement, il aime bien abréger le prédicat moderne en mimant le parler encore un peu latin de l'époque de Chrestien; prosodiquement, il se plaît à galoper impair au rythme de métal caparaçonné du décasyllabe façon Chanson de Roland. Fabriquer avec tout cela, mixé à du parler argot hypercontemporain et à une sorte de populolacanien sorti de derrière des fagots des ex-avant-gardes, une drôle de langue envers et contre tout vivante, c'est ma manie" (Prigent, 2009 : 16).

\section{RÉSUMÉS}

«La pratique poétique est d'abord pour moi un geste d'effraction dans le corps de la langue. Elle cherche à y faire craquer les coutures épidermico-sémantiques. Quand ça craque, c'est mon pari, 
du 'réel' fulgure» (Prigent, 2009 : 67). Voici les enjeux majeurs de l'écrivain et poète Christian Prigent. Il réélabore l'idée de diversité polyphonique dans le cadre d'une écriture qui soumettrait la totalité symbolique du sens en question des sens, d'un réel qui fulgure. Nous prétendons ici discuter certains fondements de son processus littéraire tenant en compte sa richesse polyphonique et translinguistique. Pourtant, la cible de notre réflexion sera la verve povera chez Prigent, c'est-à-dire son pari de mettre au jour une sorte de travail de la parole, dont la nature sensible est discours. Son écriture ne dissout pas directement les liens imaginaires et symboliques qui rendent représentables une réalité narrative, mais elle produit des effets de coupure et de discontinuité dans l'épiderme du sens à partir de ce que l'auteur appelle « un travail du négatif ». Quel serait le statut de ce travail, qui refuse d'une certaine façon la tension dialectique entre appréhension sensible et intelligibilité?

"The practices of poetry for me is a gesture of infraction in the body of the langue. It aims to break up the epidermic-semantic seams that there exists. When it breaks up, that's my bet, the real glows" (Prigent, 2009: 67). Here we have the major challenges of the writer and poet Christian Prigent. We could imagine his writings as a kind of assembly, a word-act performed by the mouth with its organicity and its prosodic unfolding. An act that is as important as the sense. Prigent reworks the idea of polyphonic diversity, framing his discourse in a literature that dismantles the symbolic totality of the sense, pluralizing it, transforming it on senses (the real that glows, as he says). We would like to discuss some fundaments of Prigent's literature, considering its trans-linguistic and polyphonic richness. And also considering his main aesthetical proposal, that is not to spare sensible nature and discourse. Prigent's literature dissolves the symbolic and imaginary bonds by which we represent a narrative experience, producing effects of discontinuity upon the epidermis of the sense. Once he recognizes a "work of the negativity" operating in the core of his literature, how could we understand it? What would be the status of this work, which refuses the dialectic tension between sensible apprehension and intelligibility?

\section{INDEX}

Mots-clés : Prigent (Christian), performance orale, voix de l'écrit, corps de la langue, négatif Keywords : Prigent (Christian), oral performance, voice of the text, body of the language, negativity

\section{AUTEUR}

\section{GUILHERME MASSARA ROCHA}

Université Fédérale de Minas Gerais

massaragr[at]gmail.com 